\title{
GAPS OF SMALLEST POSSIBLE ORDER BETWEEN PRIMES IN AN ARITHMETIC PROGRESSION
}

\author{
ROGER C. BAKER AND LIANGYI ZHAO
}

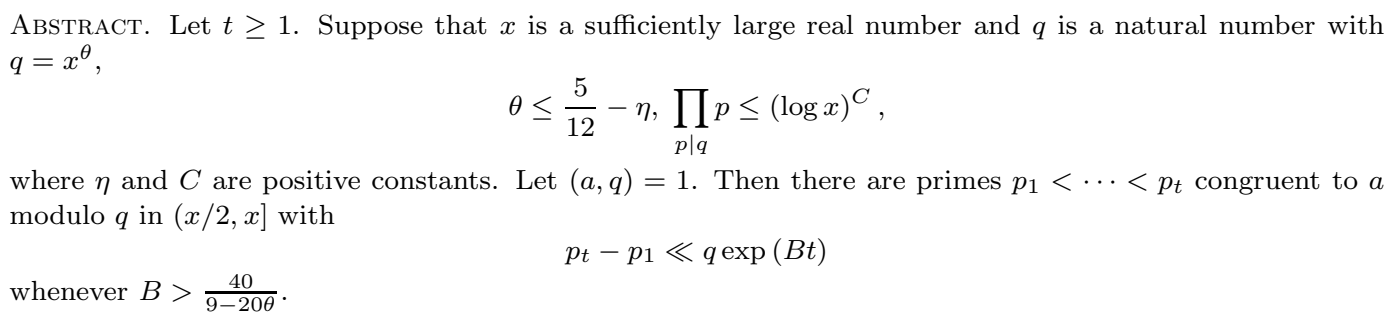

Key words and Phrases: GPY sieve, primes in arithmetic progressions, large values of Dirichlet polynomials, zeros of Dirichlet $L$-functions

2010 Mathematics Subject Classification: 11N13

\section{INTRODUCTION}

Let $t \in \mathbb{N}$ and $0 \leq \eta<1$ be given. Suppose that $x$ is a large positive real number, and that $q \in \mathbb{N}$ and $(a, q)=1, q \leq x^{1-\eta}$. Set

$$
\mathcal{A}=\{n \in(x / 2, x]: n \equiv a \quad(\bmod q)\} .
$$

It may be conjectured that there are primes $p_{1}<p_{2}<\cdots<p_{t}$ in $\mathcal{A}$ with

$$
p_{t}-p_{1} \ll_{t} q .
$$

J. Maynard [17] has recently refined the Goldston-Pintz-Yıldrım sieve to prove this in the case of $q=1$, showing that

$$
p_{t}-p_{1} \ll t^{3} \exp (4 t) \text {. }
$$

In this paper, we prove (1.1) for $q$ 's in a restricted class. Namely, we suppose that for some positive constant $\eta$ and $C$, we have $q=x^{\theta}$,

$$
\theta \leq \frac{5}{12}-\eta, \prod_{p \mid q} p \leq(\log x)^{C} .
$$

Before stating our results, we address the question of where the hypotheses in (1.2) come from. There are many striking results on the existence of primes in arithmetic progressions. D. R. Heath-Brown [12] has shown that for any $q$ and $(a, q)=1$, the least prime $p(q, a)$ congruent to $a(\bmod q)$ satisfies

$$
p(q, a) \ll q^{5.5} .
$$

G. Harman [9] has shown, subject to a weak hypothesis on the zeros of $L(s, \chi)$ for characters $\chi(\bmod q)$, that

(As usual,

$$
\pi(x ; q, a) \gg \frac{x}{\varphi(q) \log x} \text { for } q<x^{0.4736} \text { and }(a, q)=1 .
$$

$$
\left.\pi(x ; q, a)=\sum_{\substack{p \leq x \\ p \equiv a \bmod q}} 1 \text { and } \psi(x ; q, a)=\sum_{\substack{n \leq x \\ n \equiv a \bmod q}} \Lambda(n) .\right)
$$

Date: August 19, 2018. 
However, in the present paper we need a result of Bombieri-Vinogradov type in order to employ Maynard's method. For a positive constant $b$, let

$$
E_{b}(x, q)=\sum_{\substack{d \leq x^{b} \\(d, q)=1}} \max _{(a, q d)=1}\left|\psi(x ; q d, a)-\frac{x}{\varphi(q d)}\right| .
$$

We shall require a large logarithm power saving over the trivial bound for $E_{b}(x, q)$. Elliott $[8]$ achieves this for

$$
q \leq x^{1 / 3} \exp \left(-(\log \mathcal{L})^{3}\right), q=c^{n}
$$

where $c$ is a given natural number. Here and below, $\mathcal{L}:=\log x$. We weaken these restrictions, replacing them by (1.2). To do better, we would respectively need improvements of a Huxley-Jutila zero density theorem [14,16] and Siegel's theorem [6, Chapter 21].

One of our key tools, the bounds of H. Iwaniec [15] on $L$-functions, has been improved for certain ranges by M.-C. Chang [5]. This would not help us with $E_{b}(x, q)$, but see a recent paper by Banks, Freiberg and Maynard [3] for a closely related sum which requires Chang's work.

In the sequel, let $\varepsilon$ denote a positive constant sufficiently small in terms of $\eta$ and $C$.

Theorem 1. Suppose that (1.2) holds. Let

$$
L(\theta)= \begin{cases}1 / 2-\theta-\varepsilon & \text { if } \theta<2 / 5-\varepsilon, \\ 9 / 20-\theta-\varepsilon & \text { otherwise. }\end{cases}
$$

Then for $A>0$,

$$
E_{L(\theta)}(x, q) \ll \frac{x}{\varphi(q) \mathcal{L}^{A}} .
$$

The implied constant depends on $C, \theta, \varepsilon$ and $A$.

Theorem 2. Suppose that (1.2) holds. For sufficiently large $x$, there are primes $p_{1}<\cdots<p_{t}$ in $(x / 2, x]$ congruent to a $(\bmod q)$ with

$$
p_{t}-p_{1} \ll q \exp \left(\frac{2 t}{L(\theta)}\right) .
$$

The implied constant depends on $t, \eta, C$ and $\varepsilon$.

For completeness, we also include an analog of the Barban-Davenport-Halberstam theorem ( $\underline{6}$, Chapter 29]).

Theorem 3. Suppose that (1.2) holds. Let $A>0$. Then we have

$$
\sum_{\substack{d \leq Q / q \\(d, q)=1}} \sum_{\substack{a=1 \\(a, q d)=1}}^{q d}\left(\psi(x ; q d, a)-\frac{x}{\varphi(q d)}\right)^{2} \ll \frac{x Q \mathcal{L}}{\varphi(q)}
$$

whenever $x \mathcal{L}^{-A} \leq Q \leq x$. The implied constant depends on $\eta, C$ and $A$.

In what follows, we count the constant function 1 as a primitive character.

\section{Preliminary Lemmas}

Unless otherwise stated, implied constants depend on $C, \eta, \varepsilon$ and $A$ (if present).

For a Dirichlet character $\chi$, we use $\hat{\chi}$ to denote the primitive character that induces $\chi$. Moreover, let

$$
\sum_{\chi \bmod r}^{\prime} \text { and } \sum_{\chi \bmod r}^{\star}
$$


stand for, respectively, a sum restricted to nonprincipal characters modulo $r$ and a sum restricted to primitive nonprincipal characters modulo $r$. As usual, let

$$
\psi(x, \chi)=\sum_{n \leq x} \Lambda(n) \chi(n) .
$$

For a character $\chi(\bmod q d)$ where $(d, q)=1$, the conductor of $\chi$ takes the form $q_{1}(\chi) d_{1}(\chi)$ where

$$
q_{1}(\chi)\left|q, d_{1}(\chi)\right| d .
$$

Lemma 1. (i) We have, for $r<x$,

$$
\max _{(a, r)=1}\left|\psi(x ; r, a)-\frac{x}{\varphi(r)}\right| \ll \frac{1}{\varphi(r)} \sum_{\chi \bmod r}^{\prime}|\psi(x, \chi)|+\frac{x}{\varphi(r) \mathcal{L}^{A}} .
$$

(ii) For each of the characters $\chi$ in the above, we have

$$
|\psi(x, \chi)|-|\psi(x, \hat{\chi})| \ll \mathcal{L}^{2} .
$$

(iii) We have

$$
\sum_{\substack{a=1 \\(a, r)=1}}^{r}\left(\psi(x ; r, a)-\frac{x}{\varphi(r)}\right)^{2} \ll \frac{1}{\varphi(r)} \sum_{\chi \bmod r}^{\prime}|\psi(x, \chi)|^{2}+\frac{x^{2}}{\varphi(r) \mathcal{L}^{A}} .
$$

Proof. These are standard results. See, for example, pp. 162-163 and 169-170 in [6].

Lemma 2. (i) For any natural number $r$ and any complex-valued function $F$ defined on Dirichlet characters, we have

$$
\sum_{\chi \bmod r}^{\prime} F(\hat{\chi})=\sum_{r_{1} \mid r} \sum_{\chi_{1} \bmod r_{1}}^{\star} F\left(\chi_{1}\right) .
$$

(ii) Let $H>0$. Suppose further that $F \geq 0$, that $F(\hat{\chi})=0$ for $d_{1}(\chi) \leq H$, and $q D<x$. There exists $D_{1} \in(H, D]$ such that

$$
\sum_{\substack{d \leq D \\(d, q)=1}} \sum_{\chi \bmod q d}^{\prime} F(\hat{\chi}) \ll \frac{\mathcal{L} D}{D_{1}} \sum_{q_{1} \mid q} \sum_{D_{1}<d \leq 2 D_{1}} \sum_{\chi \bmod q_{1} d_{1}}^{\star} F(\chi) .
$$

Proof. The equation (2.1) is immediate from allocating the conductors of $\hat{\chi}$ into classes corresponding to divisors of $r$. For (2.2), the left-hand side is

$$
\begin{aligned}
\sum_{\substack{d \leq D \\
(d, q)=1}} \sum_{q_{1}\left|q, d_{1}\right| d} \sum_{\substack{d_{1}>H \\
\chi_{1}}}^{\star} F\left(\chi_{1}\right) & =\sum_{\substack{q_{1} \mid q \\
\bmod q_{1} d_{1}}} \sum_{\substack{H<d_{1} \leq D \\
\left(d_{1}, q\right)=1}} \sum_{\substack{d \leq D \\
(d, q)=1 \\
d \equiv 0 \bmod d_{1}}} 1 \sum_{\chi_{1} \bmod q_{1} d_{1}}^{\star} F\left(\chi_{1}\right) \\
& \ll \mathcal{L} \frac{D_{1}}{D} \sum_{q_{1} \mid q} \sum_{\substack{D_{1}<d_{1} \leq 2 D_{1} \\
\left(d_{1}, q\right)=1}} \sum_{\chi_{1} \bmod q_{1} d_{1}}^{\star} F\left(\chi_{1}\right)
\end{aligned}
$$

for some $D_{1}, H \leq D_{1} \leq D$, by splitting the range of $d_{1}$ into dyadic intervals. This completes the proof.

As an example of the last lemma, let $\varphi^{*}(r)$ denote the number of primitive characters modulo $r$. Then

$$
\sum_{r_{1} \mid r} \varphi^{*}\left(r_{1}\right)=\varphi(r)
$$

Lemma 3. Let $L=L(\theta)$ as in Theorem 1 and

$$
R(x ; r, a)=\sum_{\substack{n \leq x \\ n \equiv a \bmod r}} \Lambda(n) \log \frac{x}{n} .
$$


Suppose that for $D \ll x^{L}$ and some $A>0$,

$$
\sum_{\substack{D<d \leq 2 D \\(d, q)=1}} \max _{(a, q d)=1}\left|R(x ; q d, a)-\frac{x}{\varphi(q d)}\right| \ll \frac{x}{\varphi(q) \mathcal{L}^{2 A+1}} .
$$

Then for $D \ll x^{L}$,

$$
\sum_{\substack{D<d \leq 2 D \\(d, q)=1}} \max _{(a, q d)=1}\left|\psi(x ; q d, a)-\frac{x}{\varphi(q d)}\right| \ll \frac{x}{\varphi(q) \mathcal{L}^{A}} .
$$

Proof. We start with the identity

$$
R(x ; r, a)=\int_{1}^{x} \psi(y ; r, a) \frac{\mathrm{d} y}{y} .
$$

This, together with the fact that $\psi(y ; r, a)$ is nondecreasing in $y$, gives that for all $\lambda>0$,

$$
\begin{aligned}
\frac{R(x ; r, a)-R\left(x e^{-\lambda} ; r, a\right)}{\lambda} & =\frac{1}{\lambda} \int_{e^{-\lambda} x}^{x} \psi(y ; r, a) \frac{\mathrm{d} y}{y} \\
& \leq \psi(x ; r, a) \leq \frac{1}{\lambda} \int_{x}^{e^{\lambda} x} \psi(y ; r, a) \frac{\mathrm{d} y}{y}=\frac{R\left(e^{\lambda} x ; r, a\right)-R(x ; r, a)}{\lambda} .
\end{aligned}
$$

This leads to

$$
\psi(x ; r, a)-\frac{x}{\varphi(r)} \leq \frac{R\left(e^{\lambda} x ; r, a\right)-e^{\lambda} x / \varphi(r)}{\lambda}-\frac{R(x ; r, a)-x / \varphi(r)}{\lambda}+\left(\frac{e^{\lambda}-1}{\lambda}-1\right) \frac{x}{\varphi(r)}
$$

and

$$
\psi(x ; r, a)-\frac{x}{\varphi(r)} \geq \frac{R(x ; r, a)-x / \varphi(r)}{\lambda}-\frac{R\left(e^{-\lambda} x ; r, a\right)-e^{-\lambda} x / \varphi(r)}{\lambda}+\left(\frac{1-e^{-\lambda}}{\lambda}-1\right) \frac{x}{\varphi(r)} .
$$

Take $\lambda=\mathcal{L}^{-A-1}$ so that

$$
\frac{e^{\lambda}-1}{\lambda}-1 \ll \mathcal{L}^{-A-1} \text { and } \frac{1-e^{-\lambda}}{\lambda}-1 \ll \mathcal{L}^{-A-1} .
$$

We get, taking $D \ll x^{L}, r=q d$ and summing over $d \in(D, 2 D]$, there is $\mu \in\{1,0,-1\}$ for which

$$
\begin{aligned}
& \sum_{\substack{D<d \leq 2 D \\
(d, q)=1}} \max _{(a, q d)=1}\left|\psi(x ; q d, a)-\frac{x}{\varphi(q d)}\right| \\
& \ll \mathcal{L}^{A+1} \sum_{\substack{D<d \leq 2 D \\
(d, q)=1}} \max _{(a, q d)=1}\left|R\left(e^{\mu} x ; q d, a\right)-\frac{e^{\mu} x}{\varphi(q d)}\right|+\frac{x \mathcal{L}^{-A-1}}{\varphi(q)} \sum_{1 \leq d \leq 2 D} \frac{1}{\varphi(d)} \ll \frac{x}{\varphi(q) \mathcal{L}^{A}},
\end{aligned}
$$

using (2.4) with $e^{\mu} x$ in the place of $x$.

In the following lemma, let $\beta+i \gamma$ denote a zero of any of the Dirichlet $L$-functions $L(s, \chi)$ with $\chi$ a non-principal character modulo $r$.

Lemma 4. Let $r<x$. Then

$$
\sum_{\chi \bmod r}^{\prime}|\psi(x, \chi)| \ll \sum_{\chi \bmod r}^{\prime} \sum_{\substack{\beta \geq 1 / 2 \\|\gamma|<x^{1 / 2}}} \frac{x^{\beta} \mathcal{L}^{A+1}}{|\beta+i \gamma|^{2}}+x^{1 / 2} r \mathcal{L}^{2}+\frac{x}{\mathcal{L}^{A}} .
$$

Proof. This is a very slight variant of a result established by Elliott [8, pp. 248-249].

Let $N(\sigma, T, \chi)$ denote the number of zeros of $L(s, \chi)$ in the rectangle $[\sigma, 1) \times[-T, T]$. We shall need the following zero density result. 
Lemma 5. We have, for $T \geq 1,1 / 2 \leq \sigma<1$

$$
\sum_{\chi \bmod r}^{\prime} N(\sigma, T, \chi) \ll(r T)^{(12 / 5+\varepsilon)(1-\sigma)} .
$$

Proof. This is obtained by combining the results of M. N. Huxley [14] and M. Jutila [16].

Lemma 6. Let $a_{n}(n=1, \cdots, N)$ be complex numbers and

$$
T(\chi)=\sum_{n=1}^{N} a_{n} \chi(n) .
$$

For any natural numbers $r$ and $D$, we have

$$
\sum_{r_{1} \mid r} \sum_{\substack{d \leq D \\(d, r)=1}} \frac{r_{1} d}{\varphi\left(r_{1} d\right)} \sum_{\chi \bmod r_{1} d}^{\star}|T(\chi)|^{2} \ll\left(N+r D^{2}\right) \sum_{n=1}^{N}\left|a_{n}\right|^{2} .
$$

Proof. This is a variant of Lemma 6.5 in 7]. Set

$$
S(x)=\sum_{n=1}^{N} a_{n} e(n x)
$$

where $e(z)=\exp (2 \pi i z)$. Let

$$
\mathcal{S}=\left\{\frac{j}{d r_{1}} \in \mathbb{Q}: 1 \leq j \leq d r_{1},\left(j, d r_{1}\right)=1, d \leq D,(d, r)=1, r_{1} \mid r\right\} .
$$

It is easy to see that

$$
\left|s-s^{\prime}\right| \geq \frac{1}{r D^{2}}
$$

for all distinct $s$ and $s^{\prime}$ in $\mathcal{S}$. From the classical large sieve inequality (see [6, Chapter 27]), we get

$$
\sum_{s \in \mathcal{S}}|S(s)|^{2} \ll\left(N+r D^{2}\right) \sum_{n=1}^{N}\left|a_{n}\right|^{2} .
$$

Now by standard techniques that relate multiplicative characters to additive ones (see (10) on page 160 of [6]), we get

$$
\sum_{\chi \bmod r_{1} d}^{\star} \frac{r_{1} d}{\varphi\left(r_{1} d\right)}|T(\chi)|^{2} \leq \sum_{\substack{j=1 \\\left(j, r_{1} d\right)=1}}^{r_{1} d}\left|S\left(\frac{j}{r_{1} d}\right)\right|^{2} .
$$

Now the lemma follows by summing over pairs of $r_{1}$ and $d$ with $r_{1} \mid r$ and $d \leq D$ with $(d, r)=1$ in (2.6).

Lemma 7. Let $N \leq x, q D \leq x$ and $\mathcal{U}$ be a set of non-principal characters to moduli $q_{1} d$ with $d \leq D$, $(d, q)=1$ and $q_{1} \mid q$. Suppose that, with $T(\chi)$ as in (2.5),

$$
|T(\chi)| \geq V>0
$$

whenever $\chi \in \mathcal{U}$ and that $G=\sum_{n=1}^{N}\left|a_{n}\right|^{2}$. Then

$$
\# \mathcal{U} \ll x^{\varepsilon / 20}\left(G V^{-2} N+G^{3} V^{-6} N q D^{2}\right) .
$$

Proof. We first suppose that

$$
V>G^{1 / 2} N^{1 / 4} x^{\varepsilon / 80} .
$$

The contribution to $\# \mathcal{U}$ from a fixed $q_{1} \mid q$ is

$$
\ll x^{\varepsilon / 40}\left(G V^{-2} N+G^{3} V^{-6} N q_{1} D^{2}\right)
$$

by virtue of [14, Theorem 1]. The lemma follows on summing over $q_{1}$ with $q_{1} \mid q$.

Now suppose that

$$
V \leq G^{1 / 2} N^{1 / 4} x^{\varepsilon / 80}
$$


From Lemma 6.

$$
\# \mathcal{U} \ll G\left(N+q D^{2}\right) V^{-2} \ll G N V^{-2}+G^{3} V^{-6} N q D^{2} x^{\varepsilon / 20} .
$$

Lemma 8. For $r \geq 3$ and $T \geq 1$,

$$
\sum_{\chi \bmod }^{\star} \int_{0}^{T}\left|L\left(\frac{1}{2}+i t, \chi\right)\right|^{4} \mathrm{~d} t \ll \varphi^{*}(r) T(\log r T)^{4} .
$$

Proof. See 13] for a more precise form of this result.

Lemma 9. Let $q D<x, N \leq x,|t| \leq x^{2}$ and

$$
N(s, \chi)=\sum_{N<n \leq N^{\prime}} \chi(n) n^{-s}
$$

where $N$ and $N^{\prime}$ are natural numbers with $N^{\prime} \leq 2 N$. Then

$$
\sum_{q_{1} \mid q} \sum_{\substack{d \leq D \\(d, \bar{q})=1}} \sum_{\chi \bmod q_{1} d}^{\star}\left|N\left(\frac{1}{2}+i t, \chi\right)\right|^{4} \ll \varphi(q) D^{2} \mathcal{L}^{5}(1+|t|) .
$$

Proof. Using Perron's formula ([20, Lemma 3.12]), we see that

$$
N\left(\frac{1}{2}+i t, \chi\right)=\frac{1}{2 \pi i} \int_{1-i x^{2}}^{1+i x^{2}} L\left(\frac{1}{2}+i t+w, \chi\right)\left(\frac{\left(N^{\prime}+1 / 2\right)^{w}-(N+1 / 2)^{w}}{w}\right) \mathrm{d} w+O(1) .
$$

From the work of Heath-Brown [10], we have $L(\sigma+i t, \xi) \ll(r(|t|+1))^{3 / 16+\varepsilon}$ for $\sigma \geq 1 / 2$, where $\xi$ is a character modulo $r$. Using this bound, we can move the line of integration in (2.7) to $\left[-i x^{2}, i x^{2}\right]$ at the cost of an error of size $O(1)$. This follows from the observation that on the horizontal line segments from $\pm i x^{2}$ to $1 \pm i x^{2}$, the integrand on the right-hand side of (2.7) is

$$
\ll(q D)^{3 / 16+\varepsilon} N x^{-2+3 / 8+\varepsilon} \ll x^{9 / 16-1+2 \varepsilon} \ll 1 .
$$

Now by a splitting-up argument, it suffices to show that for $1 \leq T \leq x^{2}$ that

$$
\sum_{q_{1} \mid q} \sum_{\substack{d \leq D \\(d, q)=1}} \sum_{\bmod d q_{1}}^{\star}\left(\frac{1}{T} \int_{T-1}^{2 T}\left|L\left(\frac{1}{2}+i t+i u, \chi\right)\right| \mathrm{d} u\right)^{4} \ll \varphi(q) D^{2} \mathcal{L}^{4}(1+|t|) .
$$

By Hölder's inequality,

$$
\left(\frac{1}{T} \int_{T-1}^{2 T}\left|L\left(\frac{1}{2}+i t+i u, \chi\right)\right| \mathrm{d} u\right)^{4} \ll \frac{1}{T} \int_{T-1}^{2 T}\left|L\left(\frac{1}{2}+i t+i u, \chi\right)\right|^{4} \mathrm{~d} u .
$$

Recalling Lemma 8 and (2.3), the left-hand side of (2.8) is

$$
\begin{aligned}
& \ll \frac{1}{T} \sum_{q_{1} \mid q} \sum_{\substack{d \leq D \\
(d, \bar{q})=1}} \sum_{\chi \bmod q_{1}}^{\star} \int_{\substack{T-1+t \\
T}}^{2 T+t}\left|L\left(\frac{1}{2}+i v, \chi\right)\right|^{4} \mathrm{~d} v \\
& \ll \frac{1}{q_{1} \mid q} \sum_{\substack{d \leq D \\
(d, q)=1}} \varphi^{*}\left(q_{1} d\right) \mathcal{L}^{4}(T+|t|)=\frac{\varphi(q)}{T} \sum_{\substack{d \leq D \\
(d, q)=1}} \varphi^{*}(d) \mathcal{L}^{4}(T+|t|) .
\end{aligned}
$$

Now the lemma follows at once from this.

Next, we have the Heath-Brown decomposition of the von Mangoldt function. 
Lemma 10. Let $f(n)$ be an arbitrary complex-valued function and $k \in \mathbb{N}$. We can decompose the sum

$$
\sum_{n \leq x} \Lambda(n) f(n)
$$

into $O\left(\mathcal{L}^{2 k}\right)$ sums of the form

$$
\sum_{\substack{n_{i} \in\left[N_{i}, 2 N_{i}\right) \\ n_{1} \cdots n_{2 k} \leq x}} \log n_{1} \mu\left(n_{k+1}\right) \cdots \mu\left(n_{2 k}\right) f\left(n_{1} \cdots n_{2 k}\right)
$$

in which $N_{i} \geq 1, \prod_{i} N_{i}<x$ and $2 N_{i} \leq x^{1 / k}$ if $i>k$.

Proof. This is from [11].

Lemma 11. Suppose that (1.2) holds. Let $x \geq C_{3}(C)$ and $q \leq x$. For every primitive character $\chi(\bmod q)$, we have

$$
L(s, \chi) \neq 0, \text { if }|\Im s| \leq x, \Re s>1-\mathcal{L}^{-4 / 5}
$$

Proof. This is well-known for $q=1$ and $\chi=1$. Suppose that $q>1$. Let

$$
d=\prod_{p \mid q} p, l=\log q(x+3), \theta=\frac{1}{4 \cdot 10^{4}\left(\log d+(l \log 2 l)^{3 / 4}\right)} \geq \mathcal{L}^{-4 / 5} .
$$

According to [15, Theorem 2], there is at most one primitive character $\chi(\bmod q)$ such that there is $\rho$ with

$$
\Re \rho>1-\theta,|\Im \rho| \leq x, \text { and } L(\rho, \chi)=0 .
$$

We suppose if possible that $\chi$ exists. In this case, from [15, Theorem 2], $\chi$ is real and $\rho$ is real. From [6, Page 40], $q /(q, 8)$ is squarefree, so that $d \geq q / 8$; and from [6. page 126],

$$
\rho \leq 1-\frac{C_{4}(C)}{q^{1 /(2 C)}} \leq 1-\frac{C_{4}(C) 8^{1 /(2 C)}}{d^{1 /(2 C)}} \leq 1-\frac{C_{4}(C) 8^{1 /(2 C)}}{\mathcal{L}^{1 / 2}} .
$$

Therefore,

$$
\frac{\mathcal{L}^{1 / 2}}{C_{4}(C) 8^{1 /(2 C)}} \geq \mathcal{L}^{4 / 5}
$$

which is absurd. This completes the proof of the lemma.

\section{Proof of Theorem 1}

Let $(d, q)=1$. Given a character $\chi(\bmod q d)$ induced by $\hat{\chi}$, let $\chi^{\dagger}$ denote the character $\left(\bmod q d_{1}(\chi)\right)$ induced by $\hat{\chi}$.

Note that $\chi^{\dagger}$ shares with $\hat{\chi}$ the property

$$
\left|\psi\left(y, \chi^{\dagger}\right)\right|=|\psi(y, \chi)|+O\left(\mathcal{L}^{2}\right),(1 \leq y \leq x) .
$$

This is a consequence of Lemma 1 (ii).

Recalling Lemma 3, in order to prove Theorem 1, it remains to show that

$$
\sum_{\substack{D \leq d \leq 2 D \\(\bar{d}, q)=1}}\left|R(x ; q d, a(d))-\frac{x}{\varphi(q d)}\right| \ll \frac{x}{\varphi(q) \mathcal{L}^{2 A+3}}
$$

whenever $1 \leq D \ll X^{L(\theta)}$, for any sequence $a(d)$ with $(a(d), d q)=1$.

Now

$$
R(x ; q d, a(d))-\frac{x}{\varphi(q d)}=\frac{1}{\varphi(q d)} \sum_{\chi \bmod q d}^{\prime} \bar{\chi}(a(d)) \int_{1}^{x} \psi(y, \chi) \frac{\mathrm{d} y}{y}+O\left(\frac{x}{\varphi(q d) \mathcal{L}^{2 A+4}}\right)
$$


By replacing $\psi(y, \chi)$ by $\psi(y, \hat{\chi})$ or $\psi\left(y, \chi^{\dagger}\right)$ in (3.1), we incur an error of size

$$
\ll \mathcal{L}^{3} \ll \frac{x}{\varphi(q d) \mathcal{L}^{2 A+4}} .
$$

Therefore, it suffices to show for some absolute positive constant $C_{4}$ that

$$
\sum_{\substack{D \leq d \leq 2 D \\(d, q)=1}} \sum_{\substack{\chi \bmod q d \\ d_{1}(\chi)<\mathcal{L}^{2 A+C_{4}}}}^{\prime}\left|\int_{1}^{x} \psi\left(y, \chi^{\dagger}\right) \frac{\mathrm{d} y}{y}\right| \ll \frac{x D}{\mathcal{L}^{2 A+4}}
$$

and that

$$
\sum_{\substack{D \leq d \leq 2 D \\(d, q)=1}} \sum_{\substack{\chi \bmod q d \\ d_{1}(\chi) \geq \mathcal{L}^{2 A+C_{4}}}}^{\prime}\left|\int_{1}^{x} \psi(y, \hat{\chi}) \frac{\mathrm{d} y}{y}\right| \ll \frac{x D}{\mathcal{L}^{2 A+4}}
$$

We begin with (3.2). We observe that if $\chi^{\dagger}\left(\bmod q d_{1}\right)$ is given and $d$ is an integer divisible by $d_{1}$, then $\chi^{\dagger}$ determines $\chi(\bmod q d)$, since it is easy to see that

$$
\chi(n)=\left\{\begin{array}{cc}
\chi^{\dagger}(n) & \text { if }(n, q d)=1 \\
0 & \text { if }(n, q d)>1
\end{array}\right.
$$

Hence

$$
\begin{aligned}
\sum_{\substack{D \leq d \leq 2 D \\
(\bar{d}, q)=1}} \sum_{\substack{\chi \bmod q d \\
d_{1}(\chi)<\mathcal{L}^{2 A+C_{4}}}}^{\prime}\left|\int_{1}^{x} \psi\left(y, \chi^{\dagger}\right) \frac{\mathrm{d} y}{y}\right| & \leq \sum_{\substack{d_{1}<\mathcal{L}^{2 A+C_{4}} \\
\left(d_{1}, q\right)=1}} \sum_{\chi_{1}}^{\prime} \sum_{\bmod q d_{1}}\left|\begin{array}{c}
D \leq d<2 D \\
(d, q)=1, d_{1} \mid d
\end{array}\right| \int_{1}^{x} \psi\left(y, \chi_{1}\right) \frac{\mathrm{d} y}{y} \mid \\
& \ll \frac{\mathcal{L}^{2} D}{D_{1}} \sum_{\substack{D_{1}<d_{1} \leq 2 D_{1} \\
\left(d_{1}, q\right)=1}} \sum_{\chi_{1} \bmod q d_{1}}^{\prime}\left|\psi\left(y_{1}, \chi_{1}\right)\right|,
\end{aligned}
$$

for some $D_{1} \in\left[1, \mathcal{L}^{2 A+C_{4}}\right)$ and some $y_{1}=y_{1}(\chi), 1 \leq y_{1} \leq x$. Thus we must show that

$$
\sum_{D_{1}<d_{1} \leq 2 D_{1}} \sum_{\chi_{1}}^{\prime}\left|\psi\left(y_{1}, \chi_{1}\right)\right| \ll \frac{x D_{1}}{\mathcal{L}^{2 A+6}} .
$$

Now $q D_{1}<x^{1 / 2-\varepsilon}$. In view of Lemma 4 , with $r, A$ replaced by $q d_{1}, 2 A+6$, it suffices to show that $\left(\beta+i \gamma\right.$ denoting a zero of $\left.L\left(s, \chi_{1}\right)\right)$

$$
\sum_{\chi_{1} \bmod q d_{1}}^{\prime} \sum_{\substack{\beta>1 / 2 \\|\gamma|<x^{1 / 2}}} \frac{x^{\beta} \mathcal{L}^{2 A+7}}{|\beta+i \gamma|^{2}} \ll \frac{x}{\mathcal{L}^{2 A+6}}
$$

for each $d_{1}<\mathcal{L}^{2 A+C_{4}}$. Here, the left-hand side is

$$
\ll \mathcal{L}^{2 A+8} x^{\sigma} \sum_{\chi_{1} \bmod q d_{1}}^{\prime} \sum_{\substack{\sigma \leq \beta \leq \sigma+\mathcal{L}^{-1} \\|\gamma|<x^{1 / 2}}} \frac{1}{|\beta+i \gamma|^{2}}
$$

for some $\sigma, 1 / 2 \leq \sigma<1$. From Lemma 11] applied to $\hat{\chi}_{1}$, with $C+2 A+C_{4}$ in place of $C$, the sum in (3.4) is empty if

$$
\sigma \geq 1-\mathcal{L}^{-4 / 5}
$$

Suppose now that $\sigma<1-\mathcal{L}^{-4 / 5}$. It suffices to show that

$$
S:=\sum_{\chi_{1} \bmod q d_{1}}^{\prime} \sum_{\substack{\sigma \leq \beta<\sigma+\mathcal{L}^{-1} \\|\gamma|<x^{1 / 2}}} \frac{1}{|\beta+i \gamma|^{2}} \ll \frac{x^{1-\sigma}}{\mathcal{L}^{4 A+14}} .
$$


Using Lemma 5 ,

$$
S \ll \sum_{\chi_{1} \bmod q d_{1}}^{\prime} \sum_{j \geq 0} 2^{-2 j} N\left(\sigma, 2^{j+1}, \chi_{1}\right) \ll \sum_{j \geq 0} 2^{-j / 2}\left(q d_{1}\right)^{(12 / 5+\varepsilon)(1-\sigma)} \ll \mathcal{L}^{3 A+2 C_{4}} x^{(12 / 5+\varepsilon)(5 / 12-\eta)(1-\sigma)} .
$$

Therefore,

$$
S x^{-(1-\sigma)} \mathcal{L}^{4 A+14} \ll x^{-(12 \eta / 5-\varepsilon)(1-\sigma)} \mathcal{L}^{7 A+2 C_{4}+14} \ll \exp \left(-\left(\frac{12 \eta}{5}-\varepsilon\right) \mathcal{L}^{1 / 5}\right) \mathcal{L}^{7 A+2 C_{4}+14} \ll 1 .
$$

This completes the proof of (3.2).

For (3.3), we apply (ii) of Lemma 2. We need only show for $\mathcal{L}^{2 A+C_{4}} \leq D_{1} \leq D$ that

$$
S\left(D_{1}\right):=\sum_{q_{1} \mid q} \sum_{\substack{D_{1}<d \leq 2 D_{1} \\(d, q)=1}} \sum_{\chi \bmod q_{1} d}^{\star}\left|\int_{1}^{x} \psi(y, \chi) \frac{\mathrm{d} y}{y}\right| \ll \frac{x D_{1}}{\mathcal{L}^{2 A+5}} .
$$

For brevity, we write $\sum^{\dagger}$ in place of

$$
\sum_{q_{1} \mid q} \sum_{\substack{D_{1}<d \leq 2 D_{1} \\(d, q)=1}} \sum_{\chi \bmod q_{1} d}^{\star}
$$

Recasting the absolute value signs as coefficients, we have

$$
S\left(D_{1}\right)=\sum^{\dagger} b(\chi) \int_{1}^{x} \psi(y, \chi) \frac{\mathrm{d} y}{y}=\sum^{\dagger} b(\chi) \sum_{n \leq x} \Lambda(n) \chi(n) \log \frac{x}{n} .
$$

Now applying Lemma 10 with $k=7$ and

$$
f(n)=\sum^{\dagger} b(\chi) \chi(n) \log \frac{x}{n}
$$

we see that it suffices to show for each tuple $N_{1}, \cdots, N_{14}$ that

$$
\sum^{\dagger} b(\chi) \sum_{\substack{n_{i} \in\left(N_{i}, 2 N_{i}\right] \\ n_{1} \cdots n_{14} \leq x}} a_{1}\left(n_{1}\right) \cdots a_{14}\left(n_{14}\right) \chi\left(n_{1} \cdots n_{14}\right) \log \frac{x}{n_{1} \cdots n_{14}} \ll \frac{x D_{1}}{\mathcal{L}^{2 A+19}} .
$$

Here the coefficients $a_{j}\left(n_{j}\right)$ are those resulting from the application of Lemma 10, that is,

$$
a_{1}(n)=\log n, a_{j}(n)=1 \text { for } 2 \leq j \leq 7 \text { and } a_{j}(n)=\mu(n) \text { for } 8 \leq j \leq 14 .
$$

Using the formula

$$
\int_{1 / 2-i \infty}^{1 / 2+i \infty} y^{s} \frac{\mathrm{d} s}{s^{2}}=\left\{\begin{array}{cl}
\log y, & \text { if } y>1 \\
0, & \text { if } 0<y \leq 1
\end{array}\right.
$$

(cf. [18, p. 143]), we need to show that

$$
\sum^{\dagger} b(\chi) \int_{1 / 2-i \infty}^{1 / 2+i \infty} \sum_{\substack{n_{i} \in\left(N_{i}, 2 N_{i}\right] \\ n_{1} \cdots n_{14} \leq x}} \frac{a_{1}\left(n_{1}\right) \cdots a_{14}\left(n_{14}\right) \chi\left(n_{1} \cdots n_{14}\right)}{\left(n_{1} \cdots n_{14}\right)^{s}} \frac{x^{s} \mathrm{~d} s}{s^{2}} \ll \frac{x D_{1}}{\mathcal{L}^{2 A+19}} .
$$

Now the condition $n_{1} \cdots n_{14} \leq x$ can be removed, since the integral vanishes otherwise. We also use a trivial estimate to discard the part of the integral with $|\Im s|>x^{2}$. Thus our task is further reduced to showing that

$$
\sum^{\dagger} b(\chi) \int_{-x^{2}}^{x^{2}} N_{1}\left(\frac{1}{2}+i t, \chi\right) \cdots N_{14}\left(\frac{1}{2}+i t, \chi\right) \frac{x^{1 / 2+i t}}{(1 / 2+i t)^{2}} \mathrm{~d} t \ll \frac{x D_{1}}{\mathcal{L}^{2 A+19}},
$$


where

$$
N_{j}(s, \chi)=\sum_{N_{j}<n \leq 2 N_{j}} \frac{a_{j}(n) \chi(n)}{n^{s}} .
$$

To this end, it suffices to prove that

$$
\sum^{\dagger}\left|N_{1}\left(\frac{1}{2}+i t, \chi\right) \cdots N_{14}\left(\frac{1}{2}+i t, \chi\right)\right| \ll \frac{x^{1 / 2} D_{1}(1+|t|)}{\mathcal{L}^{2 A+20}}
$$

for $|t| \leq x^{2}$. It is convenient to recall here that $q D_{1} \ll x^{1 / 2-\varepsilon}$ for all $\theta$ and $q D_{1} \ll x^{9 / 20-\varepsilon}$ for $\theta \geq 2 / 5-\varepsilon$.

Let us write $x_{0}=\prod_{i=1}^{14} N_{i}$ and $N_{i}=x_{0}^{\alpha_{i}}$ so that $\alpha_{i} \geq 0, \alpha_{1}+\cdots+\alpha_{14}=1$ and $x_{0} \leq x$.

For a Dirichlet polynomial

$$
N(s)=\sum_{N<n \leq z N} a_{n} \chi(n) n^{-s}
$$

for some constant $z>1$, we use the abbreviation, for $p>1$,

$$
\|N\|_{p}=\left(\sum^{\dagger}\left|N\left(\frac{1}{2}+i t, \chi\right)\right|^{p}\right)^{1 / p}
$$

and

$$
\|N\|_{\infty}=\max \left\{\left|N\left(\frac{1}{2}+i t, \chi\right)\right|: \chi \text { appears in } \sum^{\dagger}\right\} .
$$

Lemma 9. possibly in conjunction with a partial summation to incorporate a $\log n$ factor, gives that

$$
\left\|N_{j}\right\|_{4}^{4} \ll q D_{1}^{2} \mathcal{L}^{9}(1+|t|) \ll D_{1} x^{1 / 2-2 \varepsilon / 3}(1+|t|)
$$

if $N_{j}>x^{1 / 6}$. If $N_{j} \leq x^{1 / 6}$, we obtain similar bounds from Lemma 6, applied to $T=N_{j}^{2}$. Indeed, in this case,

$$
\left\|N_{j}\right\|_{4}^{4} \ll\left(N_{j}^{2}+q D_{1}^{2}\right) \mathcal{L}^{4} \ll \begin{cases}D_{1} x^{1 / 2-2 \varepsilon / 3} & \text { in all cases } \\ q D_{1}^{2} \mathcal{L}^{4} & \text { if } \theta \geq 1 / 3 .\end{cases}
$$

From now on, it is convenient to arrange $N_{1}, \cdots, N_{14}$ so that

$$
N_{1} \geq \cdots \geq N_{14}
$$

The proof of (3.5) is divided into three cases.

Case 1. Suppose that $N_{1} N_{2} \geq x_{0}^{1 / 2}$. Let $M=N_{3} \cdots N_{14}$. Then the left-hand side of (3.5) is

$$
\left\|M N_{1} N_{2}\right\|_{1} \leq\|M\|_{2}\left\|N_{1}\right\|_{4}\left\|N_{2}\right\|_{4} \ll\left(M+q D_{1}^{2}\right)^{1 / 2} D_{1}^{1 / 2} x^{1 / 4-\varepsilon / 4}(1+|t|)^{1 / 2} \ll x^{1 / 4} D_{1} x^{1 / 4-\varepsilon / 4}(1+|t|)^{1 / 2} .
$$

by Hölder's inequality, Lemma $[$ and (3.6). So (3.5) holds in Case 1.

Case 2. $N_{1} N_{2}<x_{0}^{1 / 2}$ and some sub-product $\prod_{i \in \mathcal{S}} N_{i}$ (with $\mathcal{S} \subseteq\{1, \cdots, 14\}$ ) satisfies

$$
x_{0}^{1 / 2} \leq N=\prod_{i \in \mathcal{S}} N_{i}<x^{1-\theta-\varepsilon} .
$$

Hence

$$
M=\prod_{\substack{1 \leq i \leq 14 \\ i \notin \mathcal{S}}} N_{i} \leq x_{0}^{1 / 2} .
$$

The left-hand side of (3.5) is, using Lemma 6 and with $C_{4}$ suitably chosen,

$$
\|M N\|_{1} \leq\|M\|_{2}\|N\|_{2} \ll\left(M+q D_{1}^{2}\right)^{1 / 2}\left(N+q D_{1}^{2}\right)^{1 / 2} \mathcal{L}^{C_{4} / 2} \ll\left(x_{0}^{1 / 2}+q D_{1}^{2}+N^{1 / 2} q^{1 / 2} D_{1}\right) \mathcal{L}^{C_{4} / 2} .
$$

We clearly have $x_{0}^{1 / 2} \mathcal{L}^{C_{4} / 2} \ll x^{1 / 2} D_{1} \mathcal{L}^{-2 A-20}$ as $D_{1} \geq \mathcal{L}^{2 A+C_{4}}$ and $q D_{1}^{2} \mathcal{L}^{C_{4} / 2} \ll x^{1 / 2} D_{1} \mathcal{L}^{-2 A-20}$. Lastly, using (3.7),

$$
N^{1 / 2} q^{1 / 2} D_{1} \mathcal{L}^{C_{4} / 2} \ll x^{1 / 2-\varepsilon / 2} D_{1} \mathcal{L}^{C_{4} / 2} \ll x^{1 / 2} D_{1} \mathcal{L}^{-2 A-20} .
$$

So (3.5) also holds in Case 2. 
We claim that if $\theta \leq 2 / 5-\varepsilon$, then Case 1 or Case 2 must occur. Suppose not, then

$$
\alpha_{1}+\alpha_{2}<\frac{1}{2}
$$

and there is no sub-sum with

$$
\frac{2}{5} \leq \sum_{i \in \mathcal{S}} \alpha_{i} \leq \frac{3}{5}
$$

One can easily verify that this is impossible. See the details in Lemma 14 of [1].

Now we suppose that $2 / 5-\varepsilon<\theta<5 / 12$ and it still remains to consider

Case 3. $N_{1} N_{2}<x_{0}^{1 / 2}$ and no sub-product $\prod_{i \in \mathcal{S}} N_{i}$ satisfies (3.7). Since $1-\theta-\varepsilon \geq 7 / 12$ (take $\varepsilon<\eta$ ), no sub-product $\prod_{i \in \mathcal{S}} N_{i}$ lies in $\left[x_{0}^{5 / 12}, x_{0}^{7 / 12}\right]$. We start with a combinatorial lemma.

Lemma 12. Suppose that $\alpha_{1} \geq \cdots \geq \alpha_{14} \geq 0, \alpha_{1}+\cdots+\alpha_{14}=1, \alpha_{1}+\alpha_{2}<1 / 2$ and no sub-sum $\sum_{i \in \mathcal{S}} \alpha_{i}$ for a set $\mathcal{S} \subset\{1, \cdots, 14\}$ is in $[5 / 12,7 / 12]$. Then $\alpha_{5}>1 / 6$ and

$$
\alpha_{1}+\alpha_{2}+\alpha_{6}+\alpha_{7}+\cdots+\alpha_{14}<\frac{5}{12} .
$$

Proof. Clearly $\alpha_{1}+\alpha_{2}<5 / 12$. Suppose that

$$
\alpha_{1}+\alpha_{2}+\sum_{\alpha_{i} \leq 1 / 6} \alpha_{i} \geq \frac{5}{12} .
$$

Let $s$ be the least sum $\alpha_{1}+\alpha_{2}+\sum_{i \in \mathcal{B}} \alpha_{i}$, for some set $\mathcal{B} \subset\left\{i: \alpha_{i} \leq 1 / 6\right\}$, that is greater than $5 / 12$. This implies that $5 / 12 \leq s<5 / 12+1 / 6=7 / 12$, contradicting one of the conditions of the lemma. So (3.9) must be false.

We can write $\left\{i: \alpha_{i} \leq 1 / 6\right\}$ as $\{i: i>t\}$ for some $t$ with $1 \leq t \leq 14$, since the $\alpha_{i}$ 's are in descending order. If $t \geq 6$, then

which is false. If $t \leq 4$, then

$$
\alpha_{1}+\cdots+\alpha_{14} \geq \alpha_{1}+\cdots+\alpha_{t}>\frac{t}{6} \geq 1
$$

$$
\alpha_{1}+\cdots+\alpha_{14} \leq\left(\alpha_{1}+\alpha_{2}+\sum_{i>t} \alpha_{i}\right)+\left(\alpha_{3}+\alpha_{4}\right)<\frac{5}{12}+\frac{5}{12}<1
$$

which is also false. Therefore, $t=5$ and both claims of the lemma are proved.

By Lemma 12, in Case 3, we can partition $N_{1} \cdots N_{14}$ into three parts $M, N$ and $N_{5}$,

$$
M(s, \chi)=N_{1}(s, \chi) N_{2}(s, \chi) \prod_{i \geq 6} N_{i}(s, \chi)=\sum_{M \leq m \ll M} \alpha_{m} \chi(m) m^{-s}
$$

and

$$
N(s, \chi)=N_{3}(s, \chi) N_{4}(s, \chi)=\sum_{N \leq n \ll N} \beta_{n} \chi(n) n^{-s},
$$

where $M<x_{0}^{5 / 12}, N<x_{0}^{5 / 12}, N_{5}>x_{0}^{1 / 6}, M \geq N$. So $M N_{5} \geq x_{0}^{1 / 2}$.

We need the stronger assertion that

$$
N_{5}>x^{1 / 6-\varepsilon} .
$$

If this does not hold, then

$$
x_{0}^{1 / 2} \leq M N_{5}<x_{0}^{5 / 12} x^{1 / 6-\varepsilon}<x^{1-\theta-\varepsilon},
$$

an impossibility in Case 3.

The utility of (3.10) stems partly from the following lemma. 
Lemma 13. Let $\chi$ be a character modulo $q_{1} d$ that appears in $\sum^{\dagger}$. Then

$$
\sum_{k \leq K} \chi(k) \ll K^{1-\varepsilon / 2}
$$

whenever $K \geq x^{3 / 20}$.

Proof. By a theorem of D. A. Burgess [4, we have

$$
\sum_{k \leq K} \chi(n) \ll\left(q_{1} d\right)^{1 / 9+\varepsilon^{2}} K^{2 / 3} \ll x^{(9 / 20-\varepsilon)\left(1 / 9+\varepsilon^{2}\right)} K^{2 / 3} \ll K^{1-\varepsilon / 2},
$$

completing the proof.

The coefficients in $N_{5}$ cannot involve the Möbius $\mu$-function. Otherwise, from Lemma 10 and (3.10), we get

$$
x^{1 / 7} \geq 2 N_{5}>2 x^{1 / 6-\varepsilon},
$$

which is false if $\varepsilon$ is sufficiently small. Now it is easy to obtain

$$
\left\|N_{5}\right\|_{\infty} \ll N_{5}^{1 / 2} x^{-\varepsilon / 13}(1+|t|)
$$

from Lemma 13, (3.10) and a partial summation argument.

The contribution in (3.5) from $\chi$ with

$$
\min \left\{\left|M\left(\frac{1}{2}+i t, \chi\right)\right|,\left|N\left(\frac{1}{2}+i t, \chi\right)\right|,\left|N_{5}\left(\frac{1}{2}+i t, \chi\right)\right|\right\}<x^{-1}
$$

is clearly

$$
\ll \sum^{\dagger} 1 \ll x^{1 / 2-\varepsilon} D_{1} .
$$

Therefore, by a splitting-up argument, it suffices to show, for any $U, V$ and $W$ with

$$
U \leq\left\|N_{5}\right\|_{\infty}, \quad V \leq\|M\|_{\infty} \text { and } W \leq\|N\|_{\infty}
$$

that

$$
U V W \# A(U, V, W) \ll(1+|t|) x^{1 / 2} D_{1} \mathcal{L}^{-2 A-20} .
$$

Here

$$
\begin{aligned}
A(U, V, W)=\left\{\chi: \chi \text { appears in } \sum^{\dagger}, U<\left|N_{5}\left(\frac{1}{2}+i t, \chi\right)\right| \leq 2 U\right. \\
\left.\quad V<\left|M\left(\frac{1}{2}+i t, \chi\right)\right| \leq 2 V, \quad W<\left|N\left(\frac{1}{2}+i t, \chi\right)\right| \leq 2 W\right\} .
\end{aligned}
$$

Now let

$$
P=\min \left\{\frac{M+q D_{1}^{2}}{V^{2}}, \frac{N+q D_{1}^{2}}{W^{2}}, \frac{q D_{1}^{2}}{U^{4}}(1+|t|), \frac{M}{V^{2}}+\frac{q D_{1}^{2} M}{V^{6}}, \frac{N}{W^{2}}+\frac{q D_{1}^{2} N}{W^{6}}, \frac{N_{5}^{2}}{U^{4}}+\frac{q D_{1}^{2} N_{5}^{2}}{U^{12}}\right\} .
$$

It is a consequence of Lemmas [6, 7 and the first inequality in (3.6) that

$$
\# A(U, V, W) \ll P x^{\varepsilon / 20} \text {. }
$$

So it is enough to show that

$$
U V W P \ll x^{1 / 2-\varepsilon / 13} D_{1}(1+|t|) .
$$

To do this, we consider four sub-cases, according to the size of $P$ in comparison with those of $2 V^{-2} M$ and $2 W^{-2} N$.

(a) $P \leq 2 V^{-2} M$ and $P \leq 2 W^{-2} N$. In this case, (3.11) yields

$$
U V W P \ll U V W\left(V^{-2} M\right)^{1 / 2}\left(W^{-2} N\right)^{1 / 2} \ll(M N)^{1 / 2}\left\|N_{5}\right\|_{\infty} \ll x^{1 / 2-\varepsilon / 13}(1+|t|),
$$

as desired for (3.12). 
(b) $P>2 V^{-2} M$ and $P>2 W^{-2} N$. Here, we have

$$
\begin{aligned}
P \leq & 2 \min \left\{q D_{1}^{2} V^{-2}, q D_{1}^{2} W^{-2}, q D_{1}^{2} M V^{-6}, q D_{1}^{2} N W^{-6},(1+|t|) q D_{1}^{2} U^{-4}, N_{5}^{2} U^{-4}\right\} \\
& +2 \min \left\{q D_{1}^{2} V^{-2}, q D_{1}^{2} W^{-2}, q D_{1}^{2} M V^{-6}, q D_{1}^{2} N W^{-6},(1+|t|) q D_{1}^{2} U^{-4}, q D_{1}^{2} N_{5}^{2} U^{-12}\right\} \\
\leq & 2\left(q D_{1}^{2} V^{-2}\right)^{5 / 16}\left(q D_{1}^{2} W^{-2}\right)^{5 / 16}\left(q D_{1}^{2} M V^{-6}\right)^{1 / 16}\left(q D_{1}^{2} N W^{-6}\right)^{1 / 16}\left(\min \left\{q D_{1}^{2} U^{-4}, N_{5}^{2} U^{-4}\right\}\right)^{1 / 4}(1+|t|)^{1 / 4} \\
& +2 \min \left\{\left(q D_{1}^{2} V^{-2}\right)^{5 / 16}\left(q D_{1}^{2} W^{-2}\right)^{5 / 16}\left(q D_{1}^{2} M V^{-6}\right)^{1 / 16}\left(q D_{1}^{2} N W^{-6}\right)^{1 / 16}\left(q D_{1}^{2} U^{-4}\right)^{1 / 4}(1+|t|)^{1 / 4},\right. \\
\left.\left(q D_{1}^{2} V^{-2}\right)^{7 / 16}\left(q D_{1}^{2} W^{-2}\right)^{7 / 16}\left(q D_{1}^{2} M V^{-6}\right)^{1 / 48}\left(q D_{1}^{2} N W^{-6}\right)^{1 / 48}\left(q D_{1}^{2} N_{5}^{2} U^{-12}\right)^{1 / 12}\right\} & \\
\leq & 2(1+|t|)^{1 / 4}(U V W)^{-1} q D_{1}^{2}(M N)^{1 / 16}\left(\min \left\{1,\left(q D_{1}^{2}\right)^{-1 / 4} N_{5}^{1 / 2}\right\}+\min \left\{1, N_{5}^{1 / 6}(M N)^{-1 / 24}\right\}\right) \\
\ll & (1+|t|)^{1 / 4}(U V W)^{-1}\left(x^{1 / 16}\left(q D_{1}^{2}\right)^{31 / 32}+x^{1 / 20} q D_{1}^{2}\right) .
\end{aligned}
$$

Now, noting that

$$
x^{1 / 16}\left(q D_{1}^{2}\right)^{31 / 32} \ll x^{1 / 16+31 / 32 \cdot 9 / 20} D_{1}^{31 / 32} \ll x^{1 / 2-\varepsilon} D_{1}
$$

and

we get that

$$
x^{1 / 20} q D_{1}^{2} \ll x^{1 / 20} x^{9 / 20-\varepsilon} D_{1} \ll x^{1 / 2-\varepsilon} D_{1},
$$

which gives (3.12).

$$
P \ll(1+|t|)^{1 / 4}(U V W)^{-1} x^{1 / 2-\varepsilon} D_{1},
$$

(c) $P>2 V^{-2} M$ and $P \leq 2 W^{-2} N$. Now we have

$$
\begin{aligned}
P \leq & 2 \min \left\{q D_{1}^{2} V^{-2}, N W^{-2}, q D_{1}^{2} M V^{-6}, q D_{1}^{2} U^{-4}(1+|t|), N_{5}^{2} U^{-4}\right\} \\
& +2 \min \left\{q D_{1}^{2} V^{-2}, N W^{-2}, q D_{1}^{2} M V^{-6}, q D_{1}^{2} U^{-4}(1+|t|), q D_{1}^{2} N_{5}^{2} U^{-12}\right\} \\
\leq & 2\left(q D_{1}^{2} V^{-2}\right)^{1 / 8}\left(N W^{-2}\right)^{1 / 2}\left(q D_{1}^{2} M V^{-6}\right)^{1 / 8}\left(\min \left\{q D_{1}^{2} U^{-4}, N_{5}^{2} U^{-4}\right\}\right)^{1 / 4}(1+|t|)^{1 / 4} \\
& +2 \min \left\{\left(q D_{1}^{2} V^{-2}\right)^{1 / 8}\left(N W^{-2}\right)^{1 / 2}\left(q D_{1}^{2} M V^{-6}\right)^{1 / 8}\left(q D_{1}^{2} U^{-4}\right)^{1 / 4}(1+|t|)^{1 / 4},\right. \\
\left.\left(q D_{1}^{2} V^{-2}\right)^{3 / 8}\left(N W^{-2}\right)^{1 / 2}\left(q D_{1}^{2} M V^{-6}\right)^{1 / 24}\left(q D_{1}^{2} N_{5}^{2} U^{-12}\right)^{1 / 12}\right\} & \\
\leq & 2(1+|t|)^{1 / 4}(U V W)^{-1}\left(q D_{1}^{2} N\right)^{1 / 2} M^{1 / 8}\left(\min \left\{1,\left(q D_{1}^{2}\right)^{-1 / 4} N_{5}^{1 / 2}\right\}+\min \left\{1, N_{5}^{1 / 6} M^{-1 / 12}\right\}\right) \\
\ll & (1+|t|)^{1 / 4}(U V W)^{-1}\left(x^{1 / 8}\left(q D_{1}^{2}\right)^{7 / 16} N^{3 / 8}+x^{1 / 12}\left(q D_{1}^{2}\right)^{1 / 2} N^{5 / 12}\right) .
\end{aligned}
$$

To estimate these last two terms, we have

$$
x^{1 / 8}\left(q D_{1}^{2}\right)^{7 / 16} N^{3 / 8} \ll x^{1 / 8}\left(q D_{1}\right)^{7 / 16} D_{1}^{7 / 16}\left(x^{5 / 12}\right)^{3 / 8} \ll x^{1 / 8+9 / 20 \cdot 7 / 16+5 / 12 \cdot 3 / 8} D_{1}^{7 / 16} \ll x^{1 / 2-\varepsilon} D_{1}
$$

and

$$
x^{1 / 12}\left(q D_{1}^{2}\right)^{1 / 2} N^{5 / 12} \ll x^{1 / 12}\left(q D_{1}\right)^{1 / 2} D_{1}^{1 / 2} x^{25 / 144} \ll x^{1 / 12+9 / 40+25 / 144} D_{1}^{1 / 2} \ll x^{1 / 2-\varepsilon} D_{1} .
$$

These bounds lead to

giving (3.12).

$$
P \ll(1+|t|)^{1 / 4}(U V W)^{-1} x^{1 / 2-\varepsilon} D_{1},
$$

(d) $P>2 W^{-2} N$ and $P \leq 2 V^{-2} M$. We proceed the same way as in subcase (c), interchanging the roles of $M$ and $N$.

This completes the proof of Theorem 1. 


\section{Proof of Theorem 3}

From (iii) of Lemma 1, we get

$$
\sum_{\substack{d \leq Q / q \\(d, q)=1}} \sum_{\substack{a=1 \\(a, d q)=1}}^{d q}\left(\psi(x ; d q, a)-\frac{x}{\varphi(q d)}\right)^{2} \ll \sum_{\substack{d \leq Q / q \\(d, q)=1}} \frac{1}{\varphi(q d)} \sum_{\chi \bmod d q}^{\prime}|\psi(x, \chi)|^{2}+\frac{x^{2}}{\varphi(q) \mathcal{L}^{2 A}} \sum_{d \leq Q / q} \frac{1}{\varphi(d)} .
$$

As the second term is $\ll Q x \varphi(q)^{-1}$, it suffices to prove that

$$
\sum_{\substack{d \leq Q / q \\(d, q)=1}} \frac{1}{\varphi(q d)} \sum_{\chi \bmod d q}^{\prime}|\psi(x, \hat{\chi})|^{2} \ll \frac{Q x \mathcal{L}}{\varphi(q)}
$$

and that

$$
\sum_{\substack{d \leq Q / q \\(d, q)=1}} \frac{1}{\varphi(q d)} \sum_{\chi \bmod d q}^{\prime}\left(|\psi(x, \hat{\chi})|^{2}-|\psi(x, \chi)|^{2}\right) \ll \frac{Q x \mathcal{L}}{\varphi(q)} .
$$

It is easy to see that, in (4.2),

$$
|\psi(x, \hat{\chi})|^{2}-|\psi(x, \chi)|^{2} \ll\left(\sum_{p^{k} \leq x} \log p\right)\left(\sum_{p \mid d q} \log p\right) .
$$

The contribution to (4.2) from $k \geq 2$ is

$$
\ll \sum_{\substack{d \leq Q / q \\(d, q)=1}} x^{1 / 2+\varepsilon} \ll \frac{Q x^{1 / 2+\varepsilon}}{q}
$$

which is acceptable. The contribution from $k=1$ to (4.2) is

$$
\ll \sum_{\substack{d \leq Q / q \\
(d, q)=1}} x \sum_{\substack{p \leq x \\
p \mid d q}} \log p \ll x \sum_{\substack{d \leq Q / q \\
(d, q)=1}} \sum_{p \mid q} \log p+x \sum_{p \leq x} \log p \sum_{\begin{array}{c}
d \leq Q / q \\
d \equiv 0 \bmod p
\end{array}} 1 \ll \frac{x Q}{q} \log q+\frac{x Q}{q} \sum_{p \leq x} \frac{\log p}{p} \ll \frac{x Q \mathcal{L}}{\varphi(q)}
$$

which is also acceptable. (Incidentally, the error term corresponding to (4.2) is treated incorrectly on page 170 of [6]; the above discussion corrects this minor error.)

It remains to prove (4.1) in the form

$$
\sum_{q_{1} \mid q} \sum_{\substack{d_{1} \leq Q / q \\\left(d_{1}, q\right)=1}} \sum_{\substack{d \leq Q / q \\ d_{1} \mid d \\(d, q)=1}} \frac{1}{\varphi(d)} \sum_{\chi \bmod d_{1} q_{1}}^{\star}|\psi(x, \chi)|^{2} \ll Q x \mathcal{L} .
$$

We split the sum over $d_{1}$ in (4.3) into dyadic sub-sums of the form $\sum_{D<d_{1} \leq 2 D}$ where $D$ takes on the values $2^{-k} Q / q, k \geq 1$ and $2^{-k} Q / q>1 / 2$. Let $\Sigma_{D}$ denote the contribution to (4.3) from a given $D$. Hence

$$
\Sigma_{D} \ll\left(\log \frac{Q}{q D}\right) \sum_{\substack{D<d_{1} \leq 2 D \\\left(d_{1}, q\right)=1}} \frac{1}{\varphi\left(d_{1}\right)} \sum_{q_{1} \mid q} \sum_{\chi \bmod d_{1} q_{1}}^{\star}|\psi(x, \chi)|^{2} .
$$

We first deal with the contributions from $D \leq \mathcal{L}^{2 A}$ :

$$
\begin{aligned}
\sum_{D \leq \mathcal{L}^{2 A}} \Sigma_{D} & \ll \mathcal{L} x \sum_{\substack{d_{1} \leq 2 \mathcal{L}^{2 A} \\
\left(d_{1}, q\right)=1}} \frac{1}{\varphi\left(d_{1}\right)} \sum_{q_{1} \mid q} \sum_{\chi \bmod d_{1} q_{1}}^{\star}|\psi(x, \chi)| \\
& \ll \mathcal{L} x \sum_{\substack{d \leq 2 \mathcal{L}^{2 A} \\
(\bar{d}, q)=1}} \frac{1}{\varphi(d)} \sum_{\chi \bmod d q}^{\prime}\left(|\psi(x, \chi)|+\mathcal{L}^{2}\right) \ll \frac{x^{2}}{\mathcal{L}^{A}}+x \varphi(q) \mathcal{L}^{2 A+3} \ll Q x \mathcal{L},
\end{aligned}
$$


where we have used (ii) of Lemma 1 and estimates occurring in the proof of (3.2).

Now for the remaining $D$ 's with $D>\mathcal{L}^{2 A}$, we use Lemma $[$ and get

$$
\Sigma_{D} \ll \frac{1}{D} \log \frac{Q}{q D}\left(x+q D^{2}\right) \sum_{n \leq x} \Lambda^{2}(n) \ll \frac{x \mathcal{L}}{D} \log \frac{Q}{q D}\left(x+q D^{2}\right) .
$$

Now we observe easily that

$$
\sum_{D>\mathcal{L}^{2 A}} \frac{x^{2} \mathcal{L}}{D} \log \frac{Q}{q D} \ll \frac{x^{2}}{\mathcal{L}^{A}} \ll Q x
$$

and

$$
\sum_{D>\mathcal{L}^{2 A}} q x \mathcal{L} D \log \frac{Q}{q D} \ll q x \mathcal{L} \sum_{k \geq 1} k \frac{Q}{q 2^{k}} \ll Q x \mathcal{L} .
$$

This completes the proof of Theorem 3 ,

\section{Proof of Theorem 2}

We say that a set $\mathcal{H}=\left\{h_{1}, \cdots, h_{k}\right\}$ of distinct non-negative integers is admissible if for every prime $p$, there is an integer $a_{p}$ such that

$$
a_{p} \not \equiv h \quad(\bmod p)
$$

for all $h \in \mathcal{H}$.

For a set of natural numbers $\mathcal{A}$, we write $X(\mathcal{A} ; n)$ for the indicator function of $\mathcal{A}$. For a smooth function $F$ supported on

$$
\mathcal{R}_{k}=\left\{\left(x_{1}, \cdots, x_{k}\right) \in[0,1]^{k}: \sum_{i=1}^{k} x_{i} \leq 1\right\}
$$

and $1 \leq m \leq k$, let

$$
I_{k}(F)=\int_{0}^{1} \cdots \int_{0}^{1} F\left(t_{1}, \cdots, t_{k}\right)^{2} \mathrm{~d} t_{1} \cdots \mathrm{d} t_{k}
$$

and

$$
J_{k}^{(m)}(F)=\int_{0}^{1} \cdots \int_{0}^{1}\left(\int_{0}^{1} F\left(t_{1}, \cdots, t_{k}\right) \mathrm{d} t_{m}\right)^{2} \mathrm{~d} t_{1} \cdots \mathrm{d} t_{m-1} \mathrm{~d} t_{m+1} \cdots \mathrm{d} t_{k} .
$$

Furthermore, set

$$
M_{k}=\sup _{F} \frac{\sum_{m=1}^{k} J_{k}^{(m)}(F)}{I_{k}(F)},
$$

where the supremum is taken over $F$ described above with $I_{k}(F) \neq 0, J_{k}^{(m)}(F) \neq 0$ for $m=1, \cdots, k$. It is shown in [17] that

$$
M_{k} \geq \log k-2 \log \log k+O(1) .
$$

This bound is strengthened slightly in [19] to

$$
M_{k} \geq \log k+O(1)
$$

We now state a special case of [2, Theorem 1] for the integers $q$ and $a$ in the introduction. Set

$$
D_{0}=\frac{\log \log (x / 2)}{\log \log \log (x / 2)} .
$$


Lemma 14. Let $t, k$ be natural numbers and $L$ be a positive constant such that

$$
M_{k}>\frac{2 t-2}{L} \text {. }
$$

Let $\mathcal{H}=\left\{h_{1}, \cdots, h_{k}\right\}$ be an admissible set with $h_{1}<\cdots<h_{k}$, with $q \mid h_{j}$ for $j=1, \cdots, k$. Suppose that $p \mid h_{i}-h_{j}$ with $i \neq j, p>D_{0}$ implies $p \mid q$. Let $x$ be large in terms of $k$ and

Set

$$
\mathcal{A}=\left\{n: \frac{x}{2}<n \leq x, n \equiv a \quad(\bmod q)\right\} \quad \text { and } \quad \mathbb{P}=\{p: p \in \mathcal{A}\} .
$$

Suppose that

$$
Y=\frac{x}{2 q} \quad \text { and } \quad Y_{1}=\frac{1}{\varphi(q)} \int_{x / 2}^{x} \frac{\mathrm{d} t}{\log t}
$$

$$
\sum_{\substack{d \leq x^{L} \\(d, q)=1}} \mu^{2}(d) \tau_{3 k}(d)\left|\sum_{n \equiv b_{d} \bmod q d} X(\mathcal{A} ; n)-\frac{Y}{d}\right| \ll \frac{Y}{\mathcal{L}^{k+\varepsilon}}
$$

for any $b_{d} \equiv a(\bmod q)$, and

$$
\sum_{\substack{d \leq x^{L} \\(d, q)=1}} \mu^{2}(d) \tau_{3 k}(d)\left|\sum_{n \equiv b_{d} \bmod q d} X\left(\left(\mathcal{A}+h_{m}\right) \cap \mathbb{P} ; n\right)-\frac{Y_{1}}{\varphi(d)}\right| \ll \frac{Y}{\mathcal{L}^{A}}
$$

for every integer $b_{d} \equiv a(\bmod q)$ with $\left(b_{d}, q\right)=1$. Then there are primes $p_{1}<\cdots<p_{t}$ in $\mathcal{A}$ satisfying

$$
p_{t}-p_{1} \leq h_{k}-h_{1} .
$$

Proof of Theorem 2. We may suppose that $t$ is sufficiently large. Suppose that $q$ satisfies (1.2). Let

$$
\mathcal{A}=\left\{n \in\left(\frac{x}{2}, x\right]: n \equiv a \quad(\bmod q)\right\}
$$

and $0 \leq h_{1}^{\prime}<\cdots<h_{k}^{\prime}$ be an admissible set with

$$
h_{k}^{\prime} \ll k \log k \text {. }
$$

Then $\mathcal{H}=\left\{h_{1}^{\prime} q, \cdots, h_{k}^{\prime} q\right\}$ is an admissible set for which $p>D_{0}, p \mid h_{i}-h_{j}(i \neq j)$ implies $p \mid q$. Further,

$$
h_{k}^{\prime} q-h_{1}^{\prime} q \ll q k \log k \text {. }
$$

Here we choose the least $k$ such that

$$
M_{k}>\frac{2 t-2}{L(\theta)+\varepsilon / 2} .
$$

Mindful of (5.1), we get

$$
\log k \leq \frac{2 t}{L(\theta)+\varepsilon / 2}+O(1)
$$

Choosing $\varepsilon$ sufficiently small, and recalling that $t$ is large, we have

$$
\left(h_{k}^{\prime}-h_{1}^{\prime}\right) q \ll q \exp \left(\frac{2 t}{L(\theta)}\right) .
$$

It now remains to verify that the hypotheses of Lemma 14 are satisfied with $L=L(\theta)$.

The bound (5.2) presents no difficulty, as

$$
\sum_{n \equiv b_{d} \bmod d q} X(\mathcal{A} ; n)=\frac{Y}{d}+O(1) .
$$

To verify (5.3), we observe that for $(d, q)=1, b_{d} \equiv a(\bmod q)$ and $\left(b_{d}, d q\right)=1$,

$$
\sum_{n \equiv b_{d} \bmod d q} X\left(\left(\mathcal{A}+h_{m}\right) \cap \mathbb{P} ; n\right)=\sum_{\substack{p \equiv b_{d} \bmod d q \\ x / 2+h_{m}<p \leq x}} 1 .
$$


Let $A=10 k^{2}$ and

$$
R_{d}=\left|\sum_{\substack{p \equiv b_{d} \bmod d q \\ x / 2+h_{m}<p \leq x}} 1-\frac{Y_{1}}{\varphi(d)}\right| .
$$

Let $L=L(\theta)+\varepsilon / 2$. We readily deduce from Theorem 1, with $\varepsilon / 2$ in place of $\varepsilon$, that

$$
\sum_{\substack{d \leq x^{L} \\(d, q)=1}} R_{d} \ll \frac{Y}{\mathcal{L}^{A}} ;
$$

compare the argument at the end of [8]. Hence the Cauchy-Schwarz inequality together with the BrunTitchmarsh inequality gives

$$
\begin{aligned}
& \sum_{\substack{d \leq x^{L} \\
(d, q)=1}} \mu^{2}(d) \tau_{3 k}(d) R_{d} \leq\left(\sum_{\substack{d \leq x^{L} \\
(d, q)=1}} \mu^{2}(d) \tau_{3 k}^{2}(d) R_{d}\right)^{1 / 2}\left(\sum_{\substack{d \leq x^{L} \\
(d, q)=1}} R_{d}\right)^{1 / 2} \\
& \ll Y\left(\sum_{d \leq x^{L}} \frac{\tau_{3 k}^{2}(d)}{\varphi(d)}\right)^{1 / 2} \mathcal{L}^{-A / 2} \ll Y \mathcal{L}^{\left(9 k^{2}-A\right) / 2} \ll Y \mathcal{L}^{-(k+\varepsilon)} .
\end{aligned}
$$

Now we may apply Lemma 14 and obtain primes $p_{1}<\cdots<p_{t}$ in $\mathcal{A}$ with

$$
p_{t}-p_{1} \leq\left(h_{k}^{\prime}-h_{1}^{\prime}\right) q \ll q \exp \left(\frac{2 t}{L(\theta)}\right) .
$$

This completes the proof of Theorem 2

Acknowledgments. We would like to thank Tristan Freiberg for pointing out a significant defect in a previous version of this paper. This work was done while L. Z. held a visiting position in the Department of Mathematics of Brigham Young University (BYU). He wishes to thank the warm hospitality of BYU during his thoroughly enjoyable stay in Provo.

\section{REFERENCES}

[1] R. C. Baker, Primes in arithmetic progressions to spaced moduli, Acta Arith. 153 (2012), no. 2, $133-159$.

[2] R. C. Baker and L. Zhao, Bounded gaps between primes in Beatty sequences, Preprint (2014). arXiv:1411.2989.

[3] W. D. Banks, T. Freiberg, and J. Maynard, On limits points of the sequence of normalized prime gaps, Preprint (2014). arXiv: 1404.5094.

[4] D. A. Burgess, The character sum estimate with $r=3$, J. London Math. Soc. 33 (1986), no. 2, 219-226.

[5] M.-C. Chang, Short character sums for composite moduli, J. Anal. Math. 123 (2014), 1-33.

[6] H. Davenport, Multiplicative Number Theory, Third Edition, Graduate Texts in Mathematics, Springer-Verlag, New York, 2000.

[7] P. D. T. A. Elliott, Arithmetic Functions and Integer Products, Die Grundlehren der mathematischen Wissenschaften, Springer-Verlag, New York, 1985.

[8] _ Primes in progressions to moduli with a large power factor, Ramanujan J. 13 (2007), no. 1-3, 241-251.

[9] G. Harman, Watt's mean value theorem and Carmichael numbers, Int. J. Number Theory 4 (2008), no. 2, $241-248$.

[10] D. R. Heath-Brown, Hybrid bounds for Dirichlet L-functions, II, Quart. J. Math. (Oxford) Ser. (2) 31 (1980), no. 122, $157-167$.

[11] _ Prime numbers in short intervals and a generalized Vaughan identity, Canad. J. Math. 34 (1982), no. 6, 13651377.

[12] Z Z Z Z _ - free regions for Dirichlet L-functions, and the least prime in an arithmetic progression, Proc. London Math. Soc. (3) 164 (1992), no. 2, 265-338.

[13] D. R. Heath-Brown and H. M. Bui, A note on the fourth moment of Dirichlet L-functions, Acta Arith. 141 (2010), no. 4, $335-344$.

[14] M. N. Huxley, Large values of Dirichlet polynomials III, Acta Arith. 26 (1974/75), no. 4, 435-444.

[15] H. Iwaniec, On zeros of Dirichlet's L series, Invent. Math. 23 (1974), 97-104.

[16] M. Jutila, On Linnik's constant, Math. Scand. 41 (1977), 45-62.

[17] J. Maynard, Small gaps between primes, Ann. of Math. (2) 181 (2015), no. 1, 383-413. 
[18] H. L. Montgomery and R. C. Vaughan, Multiplicative Number Theory I, Classical Theory, Cambridge University Press, 2007.

[19] D. H. J. Polymath, Variants of the Selberg sieve, and bounded intervals containing many primes, Preprint (2014). arXiv: 1407.4897.

[20] E. C. Titchmarsh, The theory of the Riemann zeta-function, Second Edition, Clarendon Press, Oxford, 1986.

Roger C. Baker

Department of Mathematics

Brigham Young University

Provo, UT 84602 USA

Email: baker@math.byu.edu
Liangyi Zhao

School of Mathematics and Statistics

University of New South Wales

Sydney, NSW 2052 Australia

Email: 1.zhao@unsw.edu.au 